\title{
The Fermi Large Area Telescope: 9 years of on-orbit performance
}

\section{Robert A. Cameron*}

KIPAC/SLAC/Stanford University

E-mail: raceslac.stanford.edu

on behalf of the Fermi Large Area Telescope Collaboration

The Fermi Large Area Telescope (LAT) has been successfully operating in low Earth orbit almost continuously since its initial turn-on on 24 June 2008, for over 9 years. We present details of the current performance of the LAT detector and data acquisition sub-systems, together with long-term trends of key performance measures, and assess the expected performance in continued future operation. We also discuss the current and future status of the ground-based control, monitoring and data processing for the LAT.

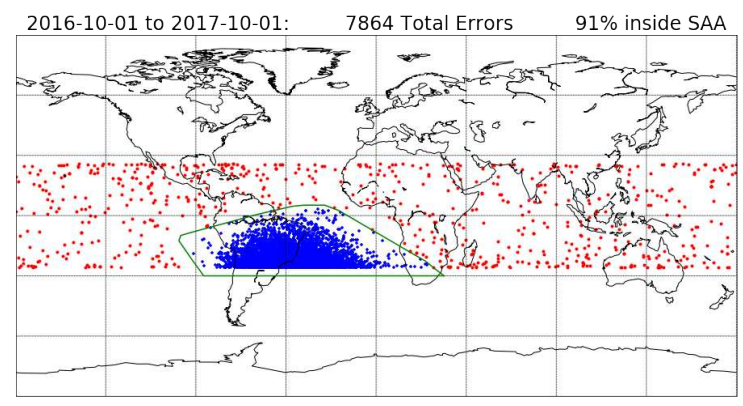

Left: Memory errors in the LAT flight computers mostly occur as Fermi passes through the South Atlantic Anomaly (SAA).

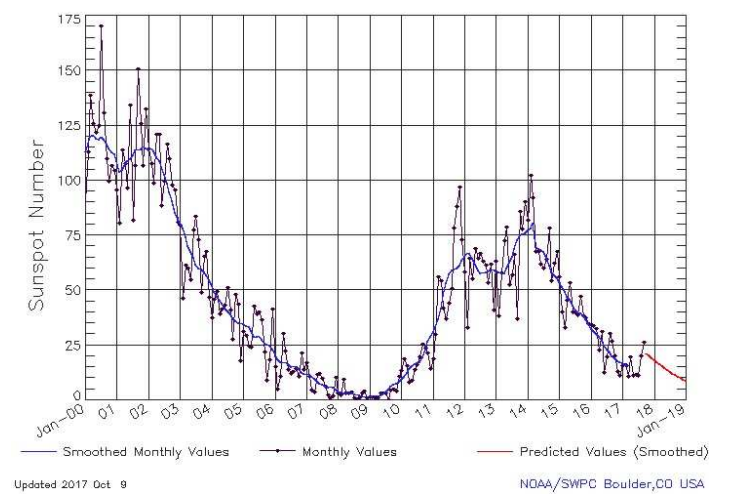

Below: The time history of LAT memory error counts in 10 Msecond periods, reflecting the Solar Cycle.

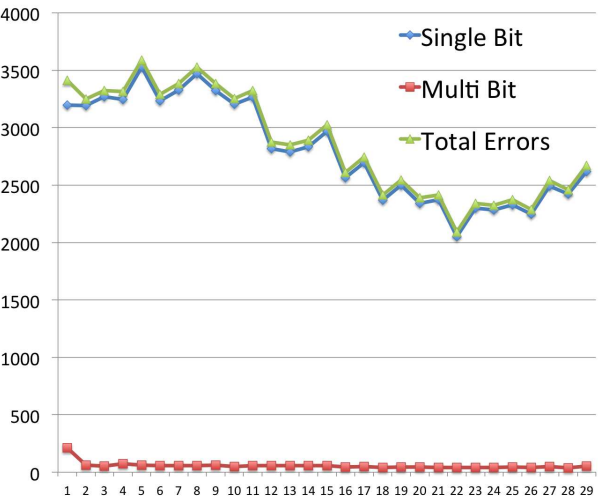

Above: Solar activity history showing the latest 12345678991011121314151617181920212223242526272829

11-year Solar Cycle.

7th Fermi Symposium 2017

15-20 October 2017

Garmisch-Partenkirchen, Germany

* Speaker. 